\title{
Physical Education and Sport in the Era of Information Technology
}

\author{
Gabibov A.B. ${ }^{1,{ }^{*}}$ Polomoshov A.F. ${ }^{1}$ Ryzhkin N.V. ${ }^{2}$
}

\author{
${ }^{1}$ Don State Agrarian University, Persianovsky village, Russia \\ ${ }^{2}$ Don State Technical University, Rostov-on-Don, Russia \\ *Corresponding author. Email: gabibovalbert@yandex.ru
}

\begin{abstract}
The article raises the issue of the introduction of information technology in the field of sports and physical education, as well as discusses new sports that arose in the information age. Information technology is being introduced into modern sports in a number of areas. One of the directions is the development of information technologies that provide the training process and the improvement of sports equipment, especially in hightech sports. Information technologies are also used in the processes of monitoring and managing the physical health of athletes and their psychological preparation for competitions. The second direction of the introduction of information technology in modern sports is the improvement of the technical and technological base of sports competitions, from equipping sports grounds and facilities to information and control systems for recording sports achievements. A separate area of sports informatization is the development of e-sports, based on modeling sports competitions using computer programs. This direction has positive aspects related to maintaining the spirit of sports competition, but also dubious moments related to the transformation of sports from a free game of physical and intellectual human forces into a kind of imitation of sports. E-sports carries the danger of turning a sports event into a kind of dehumanized competition between machines and computer program manufacturers. The active expansion of information technology into professional sports and physical education requires a separate philosophical reflection due to the fact that technical improvements pose a real threat to the significant dehumanization of modern sports and the distortion of its essence as a competition of individuals. Adherents of sports informatization seek to minimize the potential negative aspects of its informatization and actively promote e-sports competitions. Defenders of traditional sports, on the contrary, advocate restricting the informatization of sports and for decoupling the competition of machines from the competition of people in principle. An important problem of the informatization of sports and physical education is the social environment and the political system within which it is implemented. Globalization in a market economy imposes specific features of commercialization on the development of information technology. Highly informatized sport is turning into a highly profitable sector of a market economy. And this poses a problem of priority goals for the development of sports. The authors emphasize that the informatization of modern sports is generally a positive and irreversible process that requires competent political management and legal regulation.
\end{abstract}

Keywords: sports, physical education, information technology, e-sports

\section{INTRODUCTION}

The relevance of the study: in the era of information technology, physical education, sports and the organization of sports events undergo some changes. Thanks to the achievements of scientific and technological progress, not only the organization of events has improved, but also new sports have arisen. The article aims to draw attention to the technologies that have been introduced into physical education and sports, and also tells about such a modern direction of sports as e-sports.

\section{RESEARCH OBJECTIVES}

- To study the application of information technology in physical education and sports.

- Identify new information technologies in the field of sports and physical education.

- Analyze literature on the topic of the study. 
- Conduct an interview with the organizer of esports in the Don SAU.

\section{RESEARCH METHODOLOGY}

Analysis and summarization of scientific references on the topic of research, interviews.

\section{RESULTS AND DISCUSSION}

The era of information technology, which began at the end of the twentieth century, has significantly affected all areas of our lives, including sports. Physical education and sports have been enriched by various programs and information technologies, thanks to which there is facilitated monitoring of athletes' activities, training of specialists in the field of physical education is carried out, and the process of servicing sports events is facilitated.

Let's start with what information technology is.

Information technology is a combination of methods and software and technological tools that provide for the collection, storage, processing, output and use of information, which, in turn, contributes to the wide dissemination and reduces the complexity of the processes of implementation of information resources.

Information technologies in the field of sports and physical education are used in the following areas of activity:

- Improving the educational process and increasing the effectiveness of teaching.

- Management of the educational, training and organizational process in educational institutions and sports organizations, their information and methodological support.

- Automation of control, correction of the results of educational and training activities, as well as computer testing of the state of the students.

- Automation of processes for processing competition results and research.

- Organization of intellectual leisure, educational games.

- Organization of monitoring the physical condition and health of various contingents involved [3].

To date, training systems for shooting, skiing, gymnastics, physiology and some sports and pedagogical disciplines have been developed and introduced into the educational process. Using computer testing using the MMPI questionnaire allows selecting applicants and students for training in the specialty "Sports Management".

\section{RESEARCH RESULTS}

Computing technology, which has been created during this time, firmly entered the sporting life. For example, now you will not surprise anyone with digital video, digital displays, as well as measuring systems that are widely used in all major competitions.

Many modern companies produce various technical innovations that are useful for athletes.

For instance, the Finnish company FAM SPORTS has created the Check portable device, which is designed for low voltage currents and serves to record a specific neuromuscular reaction of the brain. Within 15 seconds, the device assesses the condition of the athlete and reports when the training load can lead to fatigue or injury.

Basis Science has created and launched a new bracelet watch to track human health. The watch collects and records body data during physical exertion, after which it provides the user with the information collected, thereby allowing you to track your health status as well as sports achievements.

Relatively recently, an AKSON expert system has been created that uses the results of simulation of processes in the human body during workouts. It helps to solve a number of issues that are related to the planning of training athletes, depending on the classification. Moreover, the planning can be applied both for a separate training and for long-term training [4].

Adidas has announced the so-called "smart ball" MiCoach, which can be used to train football players. This device will help in developing the kick technique and power, as well as in improving passes. Inside the smart ball there are sensors that record the necessary parameters and then transmit them via Bluetooth to a computer or smartphone [2].

C-RingDumbbells are smart dumbbells that count the number of calories lost during training time using color codes. Green means the need for further exercises, yellow means the peak of the workout, and red means to complete the workout [5].

According to literary sources, the developers of the game project Guitar Hero introduced the world a new Goji Play system, thanks to which you can combine training with a video game on the simulator. The video game runs on the iPad or iPhone, and the gadgets themselves should be fixed in the holder on the simulator. Controls in the game are only buttons on controllers and body movements that are monitored by an activity sensor. Thus, you can play sports without diverting from your favorite video game.

Now let us talk about some new sports that have arisen as a result of the introduction of modern technology in the sports field. 
Flyboard is a new water sport. Periodically, the appearance of a new device becomes the impetus for the emergence of a new sport. Something similar happened with a device called Flyboard, with which a person can literally fly over water. This sport is considered extreme and represents flying over water on a special board, which is set in motion due to the reactive power of the water flow.

Another sport that has arisen as a result of the development of technology is e-sports.

To learn more about this new sport, we turned to the organizer of e-sports in the Don SAU.

According to him, e-sports refers to team or individual competition based on video games. This type of activity can be called a sport due to the fact that it has a competitive spirit, cyber sportsmen carefully prepare before the competition, and in the process of the competition the intellectual abilities of the players are compared and certain interpersonal relationships arise between them. It is also worth noting that this activity is organized according to special rules that state what is needed to participate in the competitions, what are the restrictions, what goals are set for players, what are the criteria for e-sports players to be evaluated, and according to what principles the winner is determined.

According to the data obtained from the interview, several main classes are distinguished in e-sports, which differ in the properties of spaces, models, game task, and also developed game skills:

- First-person shooters.

- Real-time strategies.

- Sports simulators.

- Fighting.

- Team role-playing games with elements of a tactical and strategic game.

In e-sports disciplines, those video games with an element of luck and random win are not taken into account, rather the skills of tactics and strategies of esportsmen are evaluated.

It is noteworthy that at present, e-sports is indeed recognized as a separate sport, despite some differences with traditional types; it is introduced as a discipline in the educational process in schools and some universities. And today, a new sports direction is becoming increasingly popular and highly paid-in 2019 , the prize pool raffled reached \$34M [6].

Informatization of modern sports, despite the obvious positive effects and broad prospects, is an ambiguous process. This process raises discussions in the field of sports philosophy, as well as among specialists involved in sports policy issues, as well as among athletes and sports professionals themselves. In these discussions, key issues raised by the penetration of information technology into modern sports are raised.
First of all, this is the problem of the meaning of sports competition, as an agon, a competition of individuals in which they demonstrate their human qualities and the capabilities of their body. Cyber technologies in the longterm lead to elimination from sport of this main meaning and its replacement by competition of robots or computer programs, i.e. practically manufacturers of information gadgets.

The second significant problem is the problem of the effective integration of human capabilities and the capabilities of information, high-tech sports gadgets and sports equipment. A striking example of the destructive influence on the very essence of the sport of information technology is the development of chess robots that even world champions are not able to compete with.

The third problem is connected with the social status of informatization in sport, with its social goals and priorities. In a market economy, professional sports and even simple physical education are turning into branches of the economy and into an area that is far from sporting in essence and in the spirit of commercial competition between sponsors of athletes, as well as between manufacturers of sports equipment and accessories for the consumer market. In this context, sports, as such, and in particular, the informatization of sports, are not becoming a means of developing a person's personality, but a means of maximizing profit. All this distorts the essence of sport. Barry Smart in his article analyzes these points and notes, "The development of modern sport is bound up with processes of economic and cultural transformation associated with the global diffusion of capitalist forms of consumption... The global diffusion of modern sport that gathered momentum in the course of the twentieth century involved a number of networked elements, including transnational communications media and commercial corporations for which sport, especially through the iconic figure of the transnational celebrity sport star, constitutes a universally appealing globally networked cultural form. Association with sport events and sporting figures through global broadcasting, sponsorship and endorsement arrangements offers commercial corporations unique access to global consumer culture." [7]

The fourth problem is related to the legalization of informatization processes in modern sport and to the correction of sports policy in connection with these processes. Durbin, Daniel T. emphasizes, "All our sports are played in the space between the vision of order created in constitutive rules and the social conventions that establish accepted regulative rules (the rules that ultimately control play). The vision of order creates the goal. The regulative rules proscribe the acceptable behavior in achieving that goal. Regulative rules identify the penalties for failing to properly seek the goal. The two, together, create a dialectical tension that creates the compelling narrative of sport." 


\section{CONCLUSION}

Sports policy should be based on an effective combination of the interests of athletes with information technologies being introduced into sports, as well as regulate relations between consumers and manufacturers of computerized sports gadgets and technologies.

Moreover, this is obvious that in the legal regulation of the processes of modern sports informatization, the interests and health of the athletes should be with highest priority. This is especially important in the field of medical support for sports competitions and, in particular, in the fight against doping.

The fifth problem is partly technical and partly of a general worldview philosophical nature. This is the problem of the limits or boundaries of modern sports informatization. Rapid technological progress in the field of information technology opens up potentially unlimited opportunities for improving all aspects and aspects of sports competition, both in individual and team sports. But, qualitatively raising the level of sports achievements, e-sports and informatization of traditional sports are already coming to the limited capabilities of the human body as a factor that significantly limits technical progress in sports. Thus, the informatization trend faces an inevitable dilemma: either to formulate certain boundaries or limits of technical modernization in sports, especially in traditional sports, or, removing all restrictions, go to superhuman sports by modeling sports robots and sports themselves. When choosing the second perspective, sport as a human, creative, playful activity is alienated by the person himself from himself. The cost of unlimited progress is the actual dehumanization of sport. A natural question arises: does a person need endless progress at such a cost?

Thus, information technology is one of the main tools for informatization of society, including in such a field of human activity as sports and physical education. The automation process greatly facilitates the monitoring of sports events and training athletes, the organization of championships and competitions, modifies the education process for future specialists in the field of physical education and sports, and also leads to the emergence of new sports. For the most part, the introduction of new information technologies has a positive connotation; no negative influences can be traced. Although, who knows, maybe in the future - even not so far - the negative aspects of these technologies will somehow manifest themselves. The correct attitude to the processes of informatization of modern sports, obviously, does not consist in passively waiting for the possible negative consequences of this multilateral process, but in trying to anticipate them and prevent or minimize their possible dehumanistic effect. It goes without saying that the informatization of sports is an objective and irreversible process that needs, however, a competent sports policy.

\section{REFERENCES}

[1] R.S. Zhukov. New information technologies in the scientific and methodological activity of specialists in physical education and sports: status and prospects, Bulletin of the Kemerovo State University 4 (2009) 76-80.

[2] D.V. Chekasheva. Modern technologies in sports Scientific community of students of the XXI century, in: Humanities: a collection of articles based on materials of the XXXIII international student scientific-practical conference 6 (33).

[3] M.M. Shapsigov, L.S. Popova, N.B. Nart. Features of the use of information technology in the field of physical education and sports, Management of economic systems: electronic scientific journal 4 (2016) 13.

[4] M.P. Shestakov, K.B. Annenkov, E.T. Antokhina, A.V. Zubkova. Modern computer technology in the development of sports science, Theory and practice of physical education 8 (1996).

[5] New technologies in sports: education, innovation, examples, available online: https://viafuture.ru/katalog-idej/novye-tehnologii-vsporte

[6] The International 2019 prize pool exceeded \$ 34 million.

Access: https://cyber.sports.ru/dota2/1077505846.html

[7] Barry Smart Not playing around: global capitalism, modern sport and consumer culture, Global networks 7 (2) (2007) 113-114.

[8] D.T. Durbin. The Rules of Sport and the Rules of Rhetoric: Sport as Performative Public Discourse, in: Reflecting on Modern Sport in Ancient Olympia: Proceedings of the 2016 Meeting of the International Association for the Philosophy of Sport at the International Olympic Academy, edited by Heather L. Reid and Eric Moore, Fonte Aretusa, Parnassos Press, 2017. 